PI/UAN-2009-352FT

\title{
Non-gaussianity at tree and one-loop levels from vector field perturbations
}

\author{
César A. Valenzuela-Toledo ${ }^{1, *}$, Yeinzon Rodríguez ${ }^{1,2, \dagger}$ David H. Lyth ${ }^{3, \ddagger}$ \\ ${ }^{1}$ Escuela de Física, Universidad Industrial de Santander, \\ Ciudad Universitaria, Bucaramanga, Colombia \\ ${ }^{2}$ Centro de Investigaciones, Universidad Antonio Nariño, \\ Cra 3 Este \# 47A-15, Bogotá D.C., Colombia \\ ${ }^{3}$ Department of Physics, Lancaster University, \\ Lancaster $L A 1$ \& YB, UK
}

April 25, 2022

\begin{abstract}
We study the spectrum $\mathcal{P}_{\zeta}$ and bispectrum $B_{\zeta}$ of the primordial curvature perturbation $\zeta$ when the latter is generated by scalar and vector field perturbations. The tree-level and one-loop contributions from vector field perturbations are worked out considering the possibility that the one-loop contributions may be dominant over the tree-level terms (both (either) in $\mathcal{P}_{\zeta}$ and (or) in $B_{\zeta}$ ) and viceversa. The level of non-gaussianity in the bispectrum, $f_{N L}$, is calculated and related to the level of statistical anisotropy in the power spectrum, $g_{\zeta}$. For very small amounts of statistical anisotropy in the power spectrum, the level of non-gaussianity may be very high, in some cases exceeding the current observational limit.
\end{abstract}

\section{Introduction}

The anisotropies in the temperature of the cosmic microwave background (CMB) radiation, which have strong connections with the origin of the large-scale structure in the observable Universe, is one of hottest topics in modern cosmology. The properties of the CMB temperature anisotropies are described in terms of the spectral functions, like the spectrum, bispectrum, trispectrum, etc., of the primordial curvature perturbation $\zeta$ [1. In most of the cosmological models the $n$-point correlators of $\zeta$ are supposed to be translationally and rotationally invariants. However, violations of such invariances entail modifications of the usual definitions for the spectral functions in terms of the statistical descriptors [2, 3, 4. These violations may be consequences either of the presence of vector field perturbations 4, 5, 6, 7, 8, 9, 10, 11, 12, 13, 14, 15, 16, 17, 18, 19, 20, 21, 22, spinor field perturbations [23, 24], or p-form perturbations [25, 26, 27, 28, 29], contributing significantly to $\zeta$, of anisotropic expansion [10, 17, 23, 27, 30, 31, 32, 33, 34, 35] or of an inhomogeneous background [3, 4, 18. Violation of the statistical isotropy (i.e., violation of the rotational invariance in the $n$-point correlators of $\zeta$ ) seems to be present in the data [36, 37, 38, 39, 40] and, although its statistical significance is still low, the continuous presence of anomalies in every CMB data analysis (see for instance Refs. 41, 42, 43, 44, 45, 46, 47, 48, 49, 50, 51, 52, 53.) suggests the evidence might be decisive in the forthcoming years. Since the statistical anisotropy is observationally low, it entails a big problem when vector fields are present during inflation, because they generically lead to a high amount of statistical anisotropy, higher than that coming from observations [9, 10, 18. To solve this problem,

\footnotetext{
*e-mail: cavalto@ciencias.uis.edu.co

$\dagger$ e-mail: yeinzon.rodriguez@uan.edu.co

$\ddagger$ e-mail: d.lyth@lancaster.ac.uk
} 
people use different mechanisms in order to make those models consistent with observation, for example, using a triad of orthogonal vectors [4, 54, a large number of identical randomly oriented vectors fields [9, or assuming that the contribution of vector fields to the total energy density is negligible [10, 18].

Assuming statistical homogeneity of the curvature perturbation (i.e., translational invariance of the $n$-point correlators of $\zeta$ ) and pretending that during inflation the rotational invariance is broken by the presence of a vector field that points along the direction of some unit vector $\hat{\mathbf{d}}$, the most general form of the power spectrum changes from $P(k)$ to $P^{\prime}(\mathbf{k})$ : 2 ]

$$
P^{\prime}(\mathbf{k})=P(k)\left(1+g_{\zeta}(\hat{\mathbf{d}} \cdot \hat{\mathbf{k}})^{2}+\ldots\right),
$$

where $\hat{\mathbf{k}}$ is the unit vector along the direction of the wave vector $\mathbf{k}$, and $g_{\zeta}$ is the level of statistical anisotropy. The above formula gives us the primordial power spectrum that takes into account the leading effects of violations of statistical isotropy by the presence of some vector field in the inflationary era. Taking all uncertainties into account, observation is consistent with violations of the statistical isotropy at the level of $30 \%$. Recent data analysis [36, 37, 38, 39, 40] suggest the existence of violations of statistical isotropy in the five-year data from the NASA's WMAP satellite [55]. A recent study [36] of the CMB temperature perturbation finds weak evidence for statistical anisotropy; they keep only the leading (quadrupolar) term of Eq. (11):

$$
\mathcal{P}_{\zeta}(\mathbf{k})=\mathcal{P}_{\zeta}^{\text {iso }}(k)\left(1+g_{\zeta}(\hat{\mathbf{d}} \cdot \hat{\mathbf{k}})^{2}\right)
$$

and find $g_{\zeta} \simeq 0.290 \pm 0.031$ at the $68 \%$ confidence level with $\hat{\mathbf{d}}$ in a specified direction. The authors point out though that systematic uncertainties could make $g_{\zeta}$ compatible with zero. A related work [56] shows that the lowest detectable value for $\left|g_{\zeta}\right|$ from the expected performance of the NASA's WMAP satellite [55] (currently in operation) is $\left|g_{\zeta}\right| \simeq 0.1$. The same analysis gives the lowest detectable value from the expected performance of the ESA's PLANCK satellite [57]: $\left|g_{\zeta}\right| \simeq 0.02$.

As the $g_{\zeta}$ parameter has observational bounds and works, together with the non-gaussianity parameters $f_{\mathrm{NL}}$, $\tau_{\mathrm{NL}}, g_{\mathrm{NL}}$, etc., as statistical descriptors for $\zeta$, it could be a crucial tool to discriminate between some of the most usual cosmological models. In a recent paper [19, the authors point out the possibility that a vector field causes part of the primordial curvature perturbation and show that the non-gaussianity parameter $f_{\mathrm{NL}}$ is statistically anisotropic, being in principle observable. They also found a consistency relation between the parameters $g_{\zeta}$ and $f_{\mathrm{NL}}$. In such a work the authors included both vector and scalar field perturbations and kept only the lowest order terms in the expressions for the bispectrum $B_{\zeta}$ and spectrum $P_{\zeta}$. As a crucial point, they assumed that the contributions to the spectrum from vector field perturbations were smaller than those coming from scalar fields and in an opposite way for the bispectrum.

In this paper we explore other possibilities than that explored in Ref. [19, extending the analysis to include higher order contributions and studying the possibility that the loop contributions may dominate over the treelevel term:\#1. We begin our study by giving some useful formulas and calculating the 1-loop contribution to the spectrum $\mathcal{P}_{\zeta}$ and bispectrum $B_{\zeta}$ including vector and scalar fields perturbations. We then calculate the level of non-gaussianity in the bispectrum including the loop contributions and write down formulas that relate $f_{\mathrm{NL}}$ and $g_{\zeta}$. Finally, comparison with the current observational bounds for $f_{\mathrm{NL}}$ and $g_{\zeta}$ is done.

\section{Spectrum and bispectrum from vector field perturbations}

In a recent paper [18, the $\delta N$ formalism [59, 60, 61, 62, 63, was extended to include the possible statistical anisotropy in primordial curvature perturbation $\zeta$ originated from vector field perturbations. It was shown in that paper that the curvature perturbation, in the simplest case where $\zeta$ is generated by one scalar field and one vector field and assuming that the anisotropy in the expansion of the Universe is negligible, can be calculated up to quadratic terms by means of the following truncated expansion \#2

$$
\zeta(\mathbf{x}) \equiv \delta N\left(\phi(\mathbf{x}), A_{i}(\mathbf{x}), t\right)=N_{\phi} \delta \phi+N_{A}^{i} \delta A_{i}+\frac{1}{2} N_{\phi \phi}(\delta \phi)^{2}+N_{\phi A}^{i} \delta \phi \delta A_{i}+\frac{1}{2} N_{A A}^{i j} \delta A_{i} \delta A_{j},
$$

\footnotetext{
${ }^{\# 1}$ For a similar study regarding the trispectrum $T_{\zeta}$, see Ref. 58 .

\#2 This expression corrects Eq. (3.14) of Ref. 18, and Eq. (3) of Ref. 19], where a factor 2 in the fourth term of the expansion is missing.
} 
where

$$
N_{\phi} \equiv \frac{\partial N}{\partial \phi}, \quad N_{A}^{i} \equiv \frac{\partial N}{\partial A_{i}}, \quad N_{\phi \phi} \equiv \frac{\partial^{2} N}{\partial \phi^{2}}, \quad N_{A A}^{i j} \equiv \frac{\partial^{2} N}{\partial A_{i} \partial A_{j}}, \quad N_{\phi A}^{i} \equiv \frac{\partial^{2} N}{\partial A_{i} \partial \phi},
$$

$\phi$ being the scalar field and $\mathbf{A}$ the vector field, with $i$ denoting the spatial indices running from 1 to 3 . Now, we define the power spectrum $P_{\zeta}$ and the bispectrum $B_{\zeta}$ for the primordial curvature perturbation, through the Fourier modes of $\zeta$, as:

$$
\begin{aligned}
\left\langle\zeta(\mathbf{k}) \zeta\left(\mathbf{k}^{\prime}\right)\right\rangle & \equiv(2 \pi)^{3} \delta\left(\mathbf{k}+\mathbf{k}^{\prime}\right) P_{\zeta}(\mathbf{k}) \equiv(2 \pi)^{3} \delta\left(\mathbf{k}+\mathbf{k}^{\prime}\right) \frac{2 \pi^{2}}{k^{3}} \mathcal{P}_{\zeta}(\mathbf{k}) \\
\left\langle\zeta(\mathbf{k}) \zeta\left(\mathbf{k}^{\prime}\right) \zeta\left(\mathbf{k}^{\prime \prime}\right)\right\rangle & \equiv(2 \pi)^{3} \delta\left(\mathbf{k}+\mathbf{k}^{\prime}+\mathbf{k}^{\prime \prime}\right) B_{\zeta}\left(\mathbf{k}, \mathbf{k}^{\prime}, \mathbf{k}^{\prime \prime}\right) \equiv(2 \pi)^{3} \delta\left(\mathbf{k}+\mathbf{k}^{\prime}+\mathbf{k}^{\prime \prime}\right) \frac{4 \pi^{4}}{k^{3} k^{\prime 3}} \mathcal{B}_{\zeta}\left(\mathbf{k}, \mathbf{k}^{\prime}, \mathbf{k}^{\prime \prime}\right) .
\end{aligned}
$$

Using Eq. (3) and the definitions given in Eqs. (5) and (66), it was found in Ref. [18 that the tree-level contribution to the spectrum is of the form shown in Eq. (2). In addition, an analogous form for the contribution to $f_{\mathrm{NL}}$ was given in Ref. [19, showing that both, $P_{\zeta}$ and $f_{\mathrm{NL}}$ have anisotropic contributions coming from the vector field perturbation. The one-loop correction to the spectrum was also given in Ref. [18; however they kept it in an integral form. In this paper we give the loop contribution to the bispectrum, and also estimate the integrals in order to get an order of magnitude for $f_{\mathrm{NL}}$. To estimate the integrals we follow a similar procedure as that presented in Refs. 64, 65, 66, but this time including also the vector field. The expressions for $\mathcal{P}_{\zeta}$ and $\mathcal{B}_{\zeta}$, defined in Eqs. (5) and (6) and considering contributions up to one-loop order, are\#3

$$
\begin{aligned}
& \mathcal{P}_{\zeta}^{\text {tree }}(\mathbf{k})=N_{\phi}^{2} \mathcal{P}_{\delta \phi}(k)+N_{A}^{i} N_{A}^{j} \mathcal{T}_{i j}(\mathbf{k}) \\
& =N_{\phi}^{2} \mathcal{P}_{\delta \phi}(k)+N_{A}^{2} \mathcal{P}_{+}(k)+\left(\mathbf{N}_{A} \cdot \hat{\mathbf{k}}\right)^{2} \mathcal{P}_{+}(k)\left(r_{\text {long }}-1\right), \\
& \mathcal{P}_{\zeta}^{1-\text { loop }}(\mathbf{k})=\int \frac{d^{3} p k^{3}}{4 \pi|\mathbf{k}+\mathbf{p}|^{3} p^{3}}\left[\frac{1}{2} N_{\phi \phi}^{2} \mathcal{P}_{\delta \phi}(|\mathbf{k}+\mathbf{p}|) \mathcal{P}_{\delta \phi}(p)+N_{\phi A}^{i} N_{\phi A}^{j} \mathcal{P}_{\delta \phi}(|\mathbf{k}+\mathbf{p}|) \mathcal{T}_{i j}(\mathbf{p})\right. \\
& \left.+\frac{1}{2} N_{A A}^{i j} N_{A A}^{k l} \mathcal{T}_{i k}(\mathbf{k}+\mathbf{p}) \mathcal{T}_{j l}(\mathbf{p})\right], \\
& \mathcal{B}_{\zeta}^{\text {tree }}\left(\mathbf{k}, \mathbf{k}^{\prime}, \mathbf{k}^{\prime \prime}\right)=N_{\phi}^{2} N_{\phi \phi}\left[\mathcal{P}_{\delta \phi}(k) \mathcal{P}_{\delta \phi}\left(k^{\prime}\right)+\text { cyc. perm. }\right]+N_{A}^{i} N_{A}^{k} N_{A A}^{m n}\left[\mathcal{T}_{i m}(\mathbf{k}) \mathcal{T}_{k n}\left(\mathbf{k}^{\prime}\right)+\text { cyc. perm. }\right] \\
& +N_{\phi} N_{A}^{i} N_{\phi A}^{j}\left[\mathcal{P}_{\delta \phi}(k) \mathcal{T}_{i j}\left(\mathbf{k}^{\prime}\right)+5 \text { perm. }\right] \text {, } \\
& \mathcal{B}_{\zeta}^{1-\text { loop }}\left(\mathbf{k}, \mathbf{k}^{\prime}, \mathbf{k}^{\prime \prime}\right)=N_{\phi \phi}^{6} \int \frac{d^{3} p k^{3} k^{\prime 3}}{4 \pi p^{3}|\mathbf{k}+\mathbf{p}|^{3}\left|\mathbf{k}^{\prime}-\mathbf{p}\right|^{3}} \mathcal{P}_{\delta \phi}(p) \mathcal{P}_{\delta \phi}(|\mathbf{k}+\mathbf{p}|) \mathcal{P}_{\delta \phi}\left(\left|\mathbf{k}^{\prime}-\mathbf{p}\right|\right) \\
& +\quad N_{A A}^{i j} N_{A A}^{k l} N_{A A}^{m n} \int \frac{d^{3} p k^{3} k^{\prime 3}}{4 \pi p^{3}|\mathbf{k}+\mathbf{p}|^{3}\left|\mathbf{k}^{\prime}-\mathbf{p}\right|^{3}} \mathcal{T}_{i l}(\mathbf{p}) \mathcal{T}_{k n}(\mathbf{k}+\mathbf{p}) \mathcal{T}_{j m}\left(\mathbf{k}^{\prime \prime}-\mathbf{p}\right) \\
& +\quad N_{\phi \phi} N_{\phi A}^{i} N_{\phi A}^{j} \int \frac{d^{3} p k^{3} k^{\prime 3}}{4 \pi p^{3}\left|\mathbf{k}^{\prime \prime}+\mathbf{p}\right|^{3}\left|\mathbf{k}^{\prime}-\mathbf{p}\right|^{3}}\left\{\mathcal{P}_{\delta \phi}(p) \mathcal{P}_{\delta \phi}\left(\left|\mathbf{k}^{\prime \prime}+\mathbf{p}\right|\right) \mathcal{T}_{i j}\left(\mathbf{k}^{\prime}-\mathbf{p}\right)\right. \\
& \left.+\quad \mathcal{P}_{\delta \phi}(p) \mathcal{P}_{\delta \phi}\left(\left|\mathbf{k}^{\prime}-\mathbf{p}\right|\right) \mathcal{T}_{i j}\left(\mathbf{k}^{\prime \prime}+\mathbf{p}\right)+\mathcal{P}_{\delta \phi}\left(\left|\mathbf{k}^{\prime}-\mathbf{p}\right|\right) \mathcal{P}_{\delta \phi}\left(\left|\mathbf{k}^{\prime \prime}+\mathbf{p}\right|\right) \mathcal{T}_{i j}(\mathbf{p})\right\} \\
& +\quad N_{\phi A}^{i} N_{\phi A}^{j} N_{A A}^{k l} \int \frac{d^{3} p k^{3} k^{\prime 3}}{4 \pi p^{3}\left|\mathbf{k}^{\prime \prime}+\mathbf{p}\right|^{3}\left|\mathbf{k}^{\prime}-\mathbf{p}\right|^{3}}\left\{\mathcal{P}_{\delta \phi}(p) \mathcal{T}_{i k}\left(\mathbf{k}^{\prime}-\mathbf{p}\right) \mathcal{T}_{j l}\left(\mathbf{k}^{\prime \prime}+\mathbf{p}\right)\right. \\
& \left.+\mathcal{P}_{\delta \phi}\left(\left|\mathbf{k}^{\prime \prime}+\mathbf{p}\right|\right) \mathcal{T}_{i k}(\mathbf{p}) \mathcal{T}_{j l}\left(\mathbf{k}^{\prime}-\mathbf{p}\right)+\mathcal{P}_{\delta \phi}\left(\left|\mathbf{k}^{\prime}-\mathbf{p}\right|\right) \mathcal{T}_{i k}(\mathbf{p}) \mathcal{T}_{j l}\left(\mathbf{k}^{\prime \prime}+\mathbf{p}\right)\right\},
\end{aligned}
$$

where

$$
\mathcal{T}_{i j}(\mathbf{k}) \equiv T_{i j}^{\text {even }}(\mathbf{k}) \mathcal{P}_{+}(k)+i T_{i j}^{\text {odd }}(\mathbf{k}) \mathcal{P}_{-}(k)+T_{i j}^{\text {long }}(\mathbf{k}) \mathcal{P}_{\text {long }}(k)
$$

\footnotetext{
\#3 Eq. (8) corrects a mistake in Eq. (4.12) of Ref. 18 where the infinitesimal volume element $d^{3} p$ was incorrectly expressed in terms of $d p$.
} 
and

$$
T_{i j}^{\text {even }}(\mathbf{k}) \equiv \delta_{i j}-\hat{k}_{i} \hat{k}_{j}, \quad T_{i j}^{\text {odd }}(\mathbf{k}) \equiv \epsilon_{i j k} \hat{k}_{k}, \quad T_{i j}^{\text {long }}(\mathbf{k}) \equiv \hat{k}_{i} \hat{k}_{j} .
$$

Eq. (7) was written in the form of Eq. (2) with $\hat{\mathbf{d}}=\hat{\mathbf{N}}_{A}, \mathbf{N}_{A}$ being a vector with magnitude $N_{A} \equiv \sqrt{N_{A}^{i} N_{A}^{i}}$, and $r_{\text {long }} \equiv \mathcal{P}_{\text {long }} / \mathcal{P}_{+}$, where $\mathcal{P}_{\text {long }}$ is the power spectrum for the longitudinal component, and $\mathcal{P}_{+}$and $\mathcal{P}_{-}$are the parity conserving and violating power spectra defined by

$$
\mathcal{P}_{ \pm} \equiv \frac{1}{2}\left(\mathcal{P}_{R} \pm \mathcal{P}_{L}\right)
$$

with $\mathcal{P}_{R}$ and $\mathcal{P}_{L}$ denoting the power spectra for the transverse components with right-handed and left-handed polarisations [18.

The above expressions can be further separated into different terms: one due to perturbations in the scalar field, another due to the vector field perturbations, and the other due to the mixed terms:

$$
\begin{aligned}
& \mathcal{P}_{\zeta}^{\text {tree }}(\mathbf{k})=\mathcal{P}_{\zeta \phi}^{\text {tree }}(k)+\mathcal{P}_{\zeta A}^{\text {tree }}(\mathbf{k}), \\
& \mathcal{P}_{\zeta}^{1-\text { loop }}(\mathbf{k})=\mathcal{P}_{\zeta_{\phi}}^{1-\text { loop }}(k)+\mathcal{P}_{\zeta A}^{1-\text { loop }}(\mathbf{k})+\mathcal{P}_{\zeta_{\phi A}}^{1-\text { loop }}(\mathbf{k}), \\
& \mathcal{B}_{\zeta}^{\text {tree }}\left(\mathbf{k}, \mathbf{k}^{\prime}, \mathbf{k}^{\prime \prime}\right)=\mathcal{B}_{\zeta_{\phi}^{\text {tree }}}^{\text {tre }}\left(\mathbf{k}, \mathbf{k}^{\prime}, \mathbf{k}^{\prime \prime}\right)+\mathcal{B}_{\zeta}^{\text {tree }}\left(\mathbf{k}, \mathbf{k}^{\prime}, \mathbf{k}^{\prime \prime}\right)+\mathcal{B}_{\zeta_{\phi A}}^{\text {tree }}\left(\mathbf{k}, \mathbf{k}^{\prime}, \mathbf{k}^{\prime \prime}\right), \\
& \mathcal{B}_{\zeta}^{1-\text { loop }}\left(\mathbf{k}, \mathbf{k}^{\prime}, \mathbf{k}^{\prime \prime}\right)=\mathcal{B}_{\zeta \phi}^{1-\text { loop }}\left(\mathbf{k}, \mathbf{k}^{\prime}, \mathbf{k}^{\prime \prime}\right)+\mathcal{B}_{\zeta A}^{1-\text { loop }}\left(\mathbf{k}, \mathbf{k}^{\prime}, \mathbf{k}^{\prime \prime}\right)+\mathcal{B}_{\zeta \phi A}^{1-\text { loop }}\left(\mathbf{k}, \mathbf{k}^{\prime}, \mathbf{k}^{\prime \prime}\right) \text {. }
\end{aligned}
$$

Observational analysis tell us that the statistical anisotropy in the CMB temperature perturbation could be observable in a future through current experiments like WMAP or PLANCK. Eq. (2) combined with recent studies [36] tells us that the level of statistical anisotropies $g_{\zeta}$ has an upper bound and in the best case $(99 \%$ confidence level) this is $g_{\zeta} \lesssim 0.383$ [36]. During our analysis we will adopt an upper bound for $g_{\zeta}: g_{\zeta} \lesssim 0.1$. In order to satisfy the latter observational constraint over the spectrum, we must be sure that the contributions coming from vector fields in Eqs. (7) and (8) are smaller than those coming from scalar fields. That means that the first term in Eq. (14) dominates over all the other terms, even those coming from one-loop contributions. With the previous conclusion in mind we feel free to make assumptions over the other contributions, specially for those coming from vector field perturbations.

\section{Vector field contributions to the statistical descriptors}

As we explain in the previous section, our unique restriction from observation is related to the amount of statistical anisotropy present in the spectrum, so we need to be sure that the first term in Eq. (14) always dominates. In our study we will assume that the terms coming only from the vector field dominate over those coming from the mixed terms and from the scalar fields only, except for the case of the tree-level spectrum \#4. Based on the assumption made, Eqs. (14) - (17) take the form:

$$
\begin{aligned}
\mathcal{P}_{\zeta}^{\text {tree }}(\mathbf{k}) & =\mathcal{P}_{\zeta_{\phi}^{\text {tree }}}^{\text {tre }}(k)+\mathcal{P}_{\zeta}^{\text {tree }}(\mathbf{k}), \\
\mathcal{P}_{\zeta}^{1-\text { loop }}(\mathbf{k}) & =\mathcal{P}_{\zeta A}^{1-\text { loop }}(\mathbf{k}), \\
\mathcal{B}_{\zeta}^{\text {tree }}\left(\mathbf{k}, \mathbf{k}^{\prime}, \mathbf{k}^{\prime \prime}\right) & =\mathcal{B}_{\zeta A}^{\text {tree }}\left(\mathbf{k}, \mathbf{k}^{\prime}, \mathbf{k}^{\prime \prime}\right), \\
\mathcal{B}_{\zeta}^{1-\text { loop }}\left(\mathbf{k}, \mathbf{k}^{\prime}, \mathbf{k}^{\prime \prime}\right) & =\mathcal{B}_{\zeta A}^{1-\text { loop }}\left(\mathbf{k}, \mathbf{k}^{\prime}, \mathbf{k}^{\prime \prime}\right) .
\end{aligned}
$$

The above expressions lead us to four different ways that allow us to study and probably get a high level of nongaussianity $\# 5$

- Vector field spectrum $\left(\mathcal{P}_{\zeta_{A}}\right)$ and bispectrum $\left(\mathcal{B}_{\zeta_{A}}\right)$ dominated by the tree-level terms 19.

- Vector field spectrum $\left(\mathcal{P}_{\zeta_{A}}\right)$ and bispectrum $\left(\mathcal{B}_{\zeta_{A}}\right)$ dominated by the 1-loop contributions.

\footnotetext{
\#4For an actual realisation of this scenario, we need to show that such constraints are fully satisfied.

\#5 Our assumption is inspired in the one given in Ref. [65. In that work the authors use two scalar fields instead of one scalar and one vector field as in this paper. A realisation of such a scenario can be found in Refs. [1, 67] (see also Ref. 68]).
} 
- Vector field spectrum $\left(\mathcal{P}_{\zeta_{A}}\right)$ dominated by the tree-level terms and bispectrum $\left(\mathcal{B}_{\zeta_{A}}\right)$ dominated by the 1-loop contributions.

- Vector field spectrum $\left(\mathcal{P}_{\zeta_{A}}\right)$ dominated by the 1-loop contributions and bispectrum $\left(\mathcal{B}_{\zeta_{A}}\right)$ dominated by the tree-level terms.

In order to study these possibilities, we first need to estimate the integrals coming from loop contributions. From Eqs. (8), (10), (19), and (21) the integrals to solve are:

$$
\begin{aligned}
\mathcal{P}_{\zeta}{ }^{1-\text { loop }}(\mathbf{k}) & =\frac{1}{2} N_{A A}^{i j} N_{A A}^{k l} \int \frac{d^{3} p k^{3}}{4 \pi p^{3}|\mathbf{k}+\mathbf{p}|^{3}} \mathcal{T}_{i k}(\mathbf{k}+\mathbf{p}) \mathcal{T}_{j l}(\mathbf{p}), \\
\mathcal{B}_{\zeta}{ }^{1-\text { loop }}\left(\mathbf{k}, \mathbf{k}^{\prime}, \mathbf{k}^{\prime \prime}\right) & =N_{A A}^{i j} N_{A A}^{k l} N_{A A}^{m n} \int \frac{d^{3} p k^{3} k^{\prime 3}}{4 \pi p^{3}|\mathbf{k}+\mathbf{p}|^{3}\left|\mathbf{k}^{\prime}-\mathbf{p}\right|^{3}} \mathcal{T}_{i l}(\mathbf{p}) \mathcal{T}_{k n}(\mathbf{k}+\mathbf{p}) \mathcal{T}_{j m}\left(\mathbf{k}^{\prime}-\mathbf{p}\right) .
\end{aligned}
$$

The above integrals cannot be done analytically, but they can be estimated in the same way as that presented in Refs. 64, 65, 66]: these papers show that the integrals are proportional to $\ln (k L)$ where $L$ is the box size. To evaluate them we take the spectrum to be scale-invariant, which will be a good approximation if both scalar field $\phi$ and vector field $\mathbf{A}$ are sufficiently light during inflation. The integrals are logarithmically divergent at the zeros of the denominator and in each direction, but there is a cutoff at $k \sim L^{-1}$. We found that in our case the integrals are also proportional to $\ln (k L)$ and that each singularity gives equal contributions to the overall result. We find from Eqs. (22) and (23):

$$
\begin{aligned}
\mathcal{P}_{\zeta A}^{1-\text { loop }}(\mathbf{k}) & =\frac{1}{2} N_{A A}^{i j} N_{A A}^{k l}\left(2 \mathcal{P}_{+}+\mathcal{P}_{\text {long }}\right) \delta_{i k} \mathcal{T}_{j l}(\mathbf{k}) \ln (k L) \\
\mathcal{B}_{\zeta}^{1-\text { loop }}\left(\mathbf{k}, \mathbf{k}^{\prime}, \mathbf{k}^{\prime \prime}\right) & =N_{A A}^{i j} N_{A A}^{k l} N_{A A}^{m n} \ln (k L)\left(2 \mathcal{P}_{+}+\mathcal{P}_{\text {long }}\right) \delta_{i l}\left[\mathcal{T}_{k n}(\mathbf{k}) \mathcal{T}_{j m}\left(\mathbf{k}^{\prime}\right)\right]
\end{aligned}
$$

Except when considering low CMB multipoles, the box size should be set at $L \simeq H_{0} \# 6$ 68, 69, giving $\ln (k L) \sim 1$ for relevant cosmological scales.

\section{Estimating $f_{N L}$}

The non-gaussianity parameter is defined by [70, 71$] \# 7$ :

$$
f_{\mathrm{NL}}=\frac{5}{6} \frac{\mathcal{B}_{\zeta}\left(\mathbf{k}, \mathbf{k}^{\prime}, \mathbf{k}^{\prime \prime}\right)}{\left[\mathcal{P}_{\zeta}(k) \mathcal{P}_{\zeta}\left(k^{\prime}\right)+\text { cyc. perm. }\right]} .
$$

Since the isotropic contribution to the curvature perturbation is always dominant compared to the anisotropic one, we can write in the above expression only the isotropic part of the spectrum $\mathcal{P}_{\zeta}{ }^{\text {iso }}(k)$ :

$$
f_{\mathrm{NL}}=\frac{5}{6} \frac{\mathcal{B}_{\zeta}\left(\mathbf{k}, \mathbf{k}^{\prime}, \mathbf{k}^{\prime \prime}\right)}{\left[\mathcal{P}_{\zeta}^{\text {iso }}(k) \mathcal{P}_{\zeta}^{\text {iso }}\left(k^{\prime}\right)+\text { cyc. perm. }\right]} .
$$

Keeping in mind the above expression, we will estimate the possible amount of non-gaussianity generated by the anisotropic part of the primordial curvature perturbation. To do it we take into account the different possibilities mentioned in the previous section, where the non-gaussianity is produced solely by vector field perturbations.

\subsection{Vector field spectrum $\left(\mathcal{P}_{\zeta_{A}}\right)$ and bispectrum $\left(\mathcal{B}_{\zeta_{A}}\right)$ dominated by the tree-level terms}

We start our analysis by considering the case studied in Ref. [19, where the authors assume that the bispectrum is dominated by vector fields perturbations and that the higher order contributions from the vector field are always subdominant, i.e., $N_{A}^{i} \delta A_{i} \gg N_{A A}^{i j} \delta A_{i} \delta A_{j}$. This means that both the spectrum and the bispectrum are

\#6 $H_{0}$ is the Hubble parameter today.

\#7 We employ the WMAP sign convention. 
dominated by the tree-level terms, i.e., $\mathcal{P}_{\zeta}^{\text {tree }} \gg \mathcal{P}_{\zeta A}^{1-\text { loop }}$ and $\mathcal{B}_{\zeta}^{\text {tree }} \gg \mathcal{B}_{\zeta A}^{1-\text { loop }}$, so that the level of nongaussianity $f_{N L}$ is given by:

$$
f_{\mathrm{NL}}=\frac{5}{6} \frac{\mathcal{B}_{\zeta}^{\text {tree }}\left(\mathbf{k}, \mathbf{k}^{\prime}, \mathbf{k}^{\prime \prime}\right)}{\left[\mathcal{P}_{\zeta}^{\text {iso }}(k) \mathcal{P}_{\zeta}^{\text {iso }}\left(k^{\prime}\right)+\text { cyc. perm. }\right]} \simeq \frac{5}{6} \frac{\mathcal{B}_{\zeta}^{\text {tree }}\left(\mathbf{k}, \mathbf{k}^{\prime}, \mathbf{k}^{\prime \prime}\right)}{\left[\mathcal{P}_{\zeta}^{\text {iso }}(k) \mathcal{P}_{\zeta}^{\text {iso }}\left(k^{\prime}\right)+\text { cyc. perm. }\right]} .
$$

Since the anisotropic contribution to the curvature perturbation is subdominant, we can take $\mathcal{P}_{\zeta} \sim \mathcal{P}_{\zeta}$ iso, so we may write:

$$
f_{\mathrm{NL}} \simeq \frac{N_{A}^{i} N_{A}^{k} N_{A A}^{m n}\left[\mathcal{T}_{i m}(\mathbf{k}) \mathcal{T}_{k n}\left(\mathbf{k}^{\prime}\right)+\text { cyc. perm. }\right]}{\left[\mathcal{P}_{\zeta}(k) \mathcal{P}_{\zeta}\left(k^{\prime}\right)+\text { cyc. perm. }\right]}
$$

Assuming that $\mathcal{P}_{\text {long }}, \mathcal{P}_{+}$, and $\mathcal{P}_{-}$are all of the same order of magnitude, and that the spectrum is scale invariant, we may write the above equation as:

$$
f_{\mathrm{NL}} \simeq \frac{\mathcal{P}_{A}^{2} N_{A}^{2} N_{A A}}{\mathcal{P}_{\zeta}^{2}}
$$

where $\mathcal{P}_{A}=2 \mathcal{P}_{+}+\mathcal{P}_{\text {long. }}$ Taking as a typical value for the vector field perturbation $\delta A=\sqrt{\mathcal{P}_{A}}$ and $N_{A} \delta A>$ $N_{A A} \delta A^{2}$, the contribution of the vector field to $\zeta$ is given by $\zeta_{A} \sim \sqrt{\mathcal{P}_{\zeta_{A}}} \sim N_{A} \sqrt{\mathcal{P}_{A}}$. Thus, we may write an upper bound for $f_{\mathrm{NL}}$ :

$$
f_{\mathrm{NL}} \lesssim \frac{\mathcal{P}_{\zeta A}^{3 / 2}}{\mathcal{P}_{\zeta}^{2}}
$$

Since the level of statistical anisotropy in the power spectrum is of order $g_{\zeta} \sim \mathcal{P}_{\zeta_{A}} / \mathcal{P}_{\zeta}$, and since $\mathcal{P}_{\zeta}^{1 / 2} \simeq 5 \times 10^{-5}$ [72, Eq. (30) yields [19]:

$$
f_{\mathrm{NL}} \lesssim 10^{3}\left(\frac{g_{\zeta}}{0.1}\right)^{3 / 2}
$$

The above expression gives an upper bound for the level of non-gaussianity $f_{\mathrm{NL}}$ in terms of the level of statistical anisotropy in the power spectrum $g_{\zeta}$ when the former is generated by the anisotropic contribution to the curvature perturbation. As we may see, the current observational limit on $f_{N L}, f_{N L}<111$ [72], may easily be exceeded.

As an example of this model, we apply the previous results to a specific model, e.g. the vector curvaton scenario [5, 6, 7], where the $N$-derivatives are [19]:

$$
\begin{aligned}
N_{A} & =\frac{2}{3 A} r, \\
N_{A A} & =\frac{2}{A^{2}} r,
\end{aligned}
$$

where $A \equiv|\mathbf{A}|$ is the value of vector field just before the vector curvaton field decays and the parameter $r$ is the ratio between the energy density of the vector curvaton field and the total energy density of the Universe just before the vector curvaton decay. We begin exploring the conditions under which the vector field spectrum and bispectrum are always dominated by the tree-level terms. From Eqs. (7), (9), (24) and (25) our constraint leads to:

$$
\begin{aligned}
\mathcal{P}_{A} N_{A}^{2} & \gg \mathcal{P}_{A}^{2} N_{A A}^{2}, \\
\mathcal{P}_{A}^{2} N_{A}^{2} N_{A A} & \gg \mathcal{P}_{A}^{3} N_{A A}^{3} .
\end{aligned}
$$

Thus, it follows that:

$$
\mathcal{P}_{A} \ll\left(\frac{N_{A}}{N_{A A}}\right)^{2} .
$$

We have to remember that in the present case the contribution of the vector field to $\zeta$ is given by $\zeta_{A} \sim \sqrt{\mathcal{P}_{\zeta_{A}}} \sim$ $N_{A} \sqrt{\mathcal{P}_{A}}$. Then, the above equation combined with Eqs. (33) and (34) leads to:

$$
r \gg 2.25 \times 10^{-4} g_{\zeta}^{1 / 2} .
$$

This is a lower bound on the $r$ parameter we have to consider when building a realistic particle physics model of the vector curvaton scenario. 
Finally, from Eq. (30), the $f_{N L}$ parameter in this scenario is given by:

$$
f_{\mathrm{NL}} \simeq \frac{4.5 \times 10^{-2}}{r}\left(\frac{g_{\zeta}}{0.1}\right)^{2} .
$$

This is a consistency relation between $f_{N L}, g_{\zeta}$, and $r$ which will help when confronting the specific vector curvaton

realisation against observation.

\subsection{Vector field spectrum $\left(\mathcal{P}_{\zeta_{A}}\right)$ and bispectrum $\left(\mathcal{B}_{\zeta_{A}}\right)$ dominated by the 1-loop contributions}

Since the bispectrum is dominated by 1-loop contributions and is given by Eq. (25), we may write Eq. (27) as:

$$
f_{\mathrm{NL}} \simeq \frac{N_{A A}^{i j} N_{A A}^{k l} N_{A A}^{m n} \ln (k L)\left(2 \mathcal{P}_{+}+\mathcal{P}_{\text {long }}\right) \delta_{i l}\left[\mathcal{T}_{k n}(\mathbf{k}) \mathcal{T}_{j m}\left(\mathbf{k}^{\prime}\right)+\text { cyc. perm. }\right]}{\left[\mathcal{P}_{\zeta}(k) \mathcal{P}_{\zeta}\left(k^{\prime}\right)+\text { cyc. perm. }\right]} .
$$

Assuming again that $\mathcal{P}_{\text {long }}, \mathcal{P}_{+}$, and $\mathcal{P}_{-}$are all of the same order of magnitude, and that the spectrum is scale invariant, the above equation leads to:

$$
f_{\mathrm{NL}} \simeq \frac{\mathcal{P}_{A}^{3} N_{A A}^{3}}{\mathcal{P}_{\zeta}^{2}} .
$$

Since the vector field spectrum is dominated by the 1-loop contribution, $\zeta_{A} \sim \sqrt{\mathcal{P}_{\zeta_{A}}} \sim N_{A A} \mathcal{P}_{A}$. Thus, and taking into account that $g_{\zeta} \sim \mathcal{P}_{\zeta_{A}} / \mathcal{P}_{\zeta}$ and $\mathcal{P}_{\zeta}{ }^{1 / 2} \simeq 5 \times 10^{-5}$ [72], we find:

$$
f_{\mathrm{NL}} \sim \frac{1}{\sqrt{\mathcal{P}_{\zeta}}}\left(\frac{\mathcal{P}_{\zeta_{A}}}{\mathcal{P}_{\zeta}}\right)^{3 / 2} \sim 10^{3}\left(\frac{g_{\zeta}}{0.1}\right)^{3 / 2} .
$$

The biggest difference between the result found in Ref. [19], given by Eq. (32), and the result given by Eq. (42), is that the latter gives an equality relation between the non-gaussianity parameter $f_{\mathrm{NL}}$ and the level of statistical anisotropy in the power spectrum $g_{\zeta}$. Following the recent bounds for $f_{\mathrm{NL}}$ : $-9<f_{N L}<111$ [72], this scenario predicts an upper bound for the $g_{\zeta}$ parameter:

$$
g_{\zeta}<0.02 .
$$

This bound is stronger than that obtaining from direct observations in Ref. [36.

Again we apply our result to the vector curvaton scenario. Since we are assuming that the vector field spectrum and bispectrum are dominated by 1-loop contributions, we get from Eqs. (7), (9), (24), and (25):

$$
\mathcal{P}_{A}>\left(\frac{N_{A}}{N_{A A}}\right)^{2},
$$

which for the vector curvaton scenario becomes:

$$
r<2.25 \times 10^{-4} g_{\zeta}^{1 / 2}
$$

This is a lower bound on the $r$ parameter we have to consider when building a realistic particle physics model of the vector curvaton scenario.

\subsection{Vector field spectrum $\left(\mathcal{P}_{\zeta_{A}}\right)$ dominated by the tree-level terms and bispectrum $\left(\mathcal{B}_{\zeta_{A}}\right)$ dominated by the 1-loop contributions}

In order to check the viability of this case, we start studying the implications of the restrictions over the spectrum and the bispectrum, i.e., what happens when we assume that the vector field spectrum is dominated by the treelevel terms and the bispectrum is dominated by the 1-loop contributions. From Eqs. (7), (99), (24), and (25) it follows that:

$$
\begin{gathered}
\mathcal{P}_{A} N_{A}^{2} \gg \mathcal{P}_{A}^{2} N_{A A}^{2} \quad \Rightarrow \quad \mathcal{P}_{A} \ll \frac{N_{A}^{2}}{N_{A A}^{2}}, \\
\mathcal{P}_{A}^{2} N_{A}^{2} N_{A A} \ll \mathcal{P}_{A}^{3} N_{A A}^{3} \quad \Rightarrow \quad \mathcal{P}_{A} \gg \frac{N_{A}^{2}}{N_{A A}^{2}} .
\end{gathered}
$$


As we may see, it is impossible to satisfy simultaneously Eqs. (46) and (47). This is perhaps related to the fact that we have taken into account only one vector field. Such a conclusion may be relaxed if we take into account more than one vector field, as analogously happens in the scalar multifield case [1, 67.

\subsection{Vector field spectrum $\left(\mathcal{P}_{\zeta_{A}}\right)$ dominated by the 1-loop contributions and bispec- trum $\left(\mathcal{B}_{\zeta_{A}}\right)$ dominated by the tree-level terms}

As in the previous case, it is impossible to satisfy the conditions under which the spectrum is always dominated by the 1-loop contributions and the bispectrum is always dominated by the tree-level terms:

$$
\begin{aligned}
\mathcal{P}_{A} \gg \frac{N_{A}^{2}}{N_{A A}^{2}}, \\
\mathcal{P}_{A} \ll \frac{N_{A}^{2}}{N_{A A}^{2}} .
\end{aligned}
$$

Again, the conclusion may be relaxed if we take into account more than one vector field.

\section{Conclusions}

We have studied in this paper the order of magnitude of the level of non-gaussianity in the bispectrum $f_{N L}$ when statistical anisotropy is generated by the presence of one vector field. Particularly, we have shown that it is possible to get a high level of non-gaussianity if we assume that the 1-loop contributions dominate over the tree-level terms in both the vector field spectrum $\left(\mathcal{P}_{\zeta_{A}}\right)$ and the bispectrum $\left(\mathcal{B}_{\zeta_{A}}\right) . f_{\mathrm{NL}}$ is given in this case by Eq. (42), where we may see that there is a consistency relation between $f_{\mathrm{NL}}$ and the amount of statistical anisotropy in the spectrum $g_{\zeta}$. Such a consistency relation lets us fix one of the two parameters, i.e., if the nongaussianity in the bispectrum is detected and our scenario is appropriate, the amount of statistical anisotropy in the power spectrum must have a specific value, which is given by Eq. (42). A similar conclusion is reached if the statistical anisotropy in the power spectrum is detected before the non-gaussianity in the bispectrum is. As an example we may see the result given in Eq. (43), where an indirect (but stronger than the observational) upper bound on $g_{\zeta}$ is obtaining from the current upper observational bound on $f_{\mathrm{NL}}$.

\section{Acknowledgments}

This work was supported by STFC grant ST/G000549/1 and by EU grant MRTN-CT-2006-035863. C.A.V.-T. acknowledges the Department of Physics at Lancaster University in Lancaster (UK) were part of this work was done.

\section{References}

[1] H. R. S. Cogollo, Y. Rodríguez, and C. A. Valenzuela-Toledo, On the issue of the $\zeta$ series convergence and loop corrections in the generation of observable primordial non-gaussianity in slow-roll inflation. Part I: the bispectrum, JCAP 0808, 029 (2008).

[2] L. Ackerman, S. M. Carroll, and M. B. Wise, Imprints of a primordial preferred direction on the microwave background, Phys. Rev. D 75, 083502 (2007).

[3] S. M. Carroll, C.-Y. Tseng, and M. B. Wise, Translational invariance and the anisotropy of the cosmic microwave background, arXiv:0811.1086 [astro-ph].

[4] C. Armendariz-Picon, Creating statistically anisotropic and inhomogeneous perturbations, JCAP 0709, 014 (2007).

[5] K. Dimopoulos, Can a vector field be responsible for the curvature perturbation in the Universe?, Phys. Rev. D 74, $083502(2006)$. 
[6] K. Dimopoulos, Supergravity inspired vector curvaton, Phys. Rev. D 76, 063506 (2007).

[7] K. Dimopoulos and M. Karčiauskas, Non-minimally coupled vector curvaton, JHEP 0807, 119 (2008).

[8] S. Yokoyama and J. Soda, Primordial statistical anisotropy generated at the end of inflation, JCAP 0808, 005 (2008).

[9] A. Golovnev, V. Mukhanov, and V. Vanchurin, Vector inflation, JCAP 0806, 009 (2008).

[10] S. Kanno, M. Kimura, J. Soda, and S. Yokoyama, Anisotropic inflation from vector impurity, JCAP 0808, 034 (2008).

[11] A. Golovnev, V. Mukhanov, and V. Vanchurin, Gravitational waves in vector inflation, JCAP 0811, 018 (2008).

[12] A. Golovnev and V. Vanchurin, Cosmological perturbations from vector inflation, Phys. Rev. D 79, 103524 (2009).

[13] S. Koh and B. Hu, Timelike vector field dynamics in the early Universe, arXiv:0901.0429 [hep-th].

[14] B. Himmetoglu, C. R. Contaldi, and M. Peloso, Instability of the Ackerman-Carroll-Wise model, and problems with massive vectors during inflation, Phys. Rev. D 79, 063517 (2009).

[15] B. Himmetoglu, C. R. Contaldi, and M. Peloso, Instability of anisotropic cosmological solutions supported by vector fields, Phys. Rev. Lett. 102, 111301 (2009).

[16] B. Himmetoglu, C. R. Contaldi, and M. Peloso, Ghost instabilities of cosmological models with vector fields nonminimally coupled to the curvature, arXiv:0909.3524 [astro-ph.CO].

[17] B. Himmetoglu, Spectrum of perturbations in anisotropic inflationary Universe with vector hair, arXiv:0910.3235 [astro-ph.CO].

[18] K. Dimopoulos, M. Karčiauskas, D. H. Lyth, and Y. Rodríguez, Statistical anisotropy of the curvature perturbation from vector field perturbations, JCAP 0905, 013 (2009).

[19] M. Karčiauskas, K. Dimopoulos, and D. H. Lyth, Anisotropic non-gaussianity from vector field perturbations, Phys. Rev. D 80, 023509 (2009).

[20] K. Dimopoulos, M. Karčiauskas, and J. M. Wagstaff, Vector curvaton with varying kinetic function, arXiv:0907.1838 [hep-ph].

[21] N. Bartolo, E. Dimastrogiovanni, S. Matarrese, and A. Riotto, Anisotropic bispectrum of curvature perturbations from primordial non-Abelian vector fields, JCAP 0910, 015 (2009).

[22] K. Dimopoulos, M. Karčiauskas, and J. M. Wagstaff, Vector curvaton without instabilities, arXiv:0909.0475 [hep-ph].

[23] C. G. Böhmer and D. F. Mota, CMB anisotropies and inflation from non-standard spinors, Phys. Lett. B 663, 168 (2008).

[24] S. Shankaranarayanan, What-if inflaton is a spinor condensate?, arXiv:0905.2573 [astro-ph.C0].

[25] C. Germani and A. Kehagias, P-nflation: generating cosmic inflation with p-forms, JCAP 0903, 028 (2009).

[26] T. Kobayashi and S. Yokoyama, Gravitational waves from p-form inflation, JCAP 0905, 004 (2009).

[27] T. S. Koivisto, D. F. Mota, and C. Pitrou, Inflation from n-forms and its stability, JHEP 0909, 092 (2009).

[28] C. Germani and A. Kehagias, Scalar perturbations in p-nflation: the 3-form case, JCAP 0911,005 (2009).

[29] T. S. Koivisto and N. J. Nunes, Inflation and dark energy from three-forms, arXiv:0908.0920 [astro-ph.CO]. 
[30] T. S. Pereira, C. Pitrou, and J.-P. Uzan, Theory of cosmological perturbations in an anisotropic Universe, JCAP 0709, 006 (2007).

[31] A. E. Gumrukcuoglu, C. R. Contaldi, and M. Peloso, Inflationary perturbations in anisotropic backgrounds and their imprint on the cosmic microwave background, JCAP 0711, 005 (2007).

[32] C. Pitrou, T. S. Pereira, and J.-P. Uzan, Predictions from an anisotropic inflationary era, JCAP 0804, 004 (2008).

[33] M.-a. Watanabe, S. Kanno, and J. Soda, Inflationary Universe with anisotropic hair, Phys. Rev. Lett. 102, $191302(2009)$.

[34] K. Bamba and S. Nojiri, Cosmology in non-minimal Yang-Mills/Maxwell theory, arXiv:0811.0150 [hep-th].

[35] P.-P. Dechant, A. N. Lasenby, and M. P. Hobson, An anisotropic, non-singular early Universe model leading to a realistic cosmology, Phys. Rev. D 79, 043524 (2009).

[36] N. E. Groeneboom, L. Ackerman, I. K. Wehus, and H. K. Eriksen, Bayesian analysis of an anisotropic universe model: systematics and polarization, arXiv:0911.0150 [astro-ph.C0].

[37] N. E. Groeneboom and H. K. Eriksen, Bayesian analysis of sparse anisotropic universe models and application to the 5-yr WMAP data, Astrophys. J. 690, 1807 (2009).

[38] C. Armendariz-Picon and L. Pekowsky, Bayesian limits on primordial isotropy breaking, Phys. Rev. Lett. 102, 031301 (2009).

[39] D. Hanson and A. Lewis, Estimators for CMB statistical anisotropy, Phys. Rev. D 80, 063004 (2009).

[40] P. K. Samal, R. Saha, P. Jain, and J. P. Ralston, Signals of statistical anisotropy in WMAP foregroundcleaned maps, Mon. Not. R. Astron. Soc. 396, 511 (2009).

[41] Z. Hou et. al., Frequentist comparison of CMB local extrema statistics in the five-year WMAP data with two anisotropic cosmological models, arXiv:0910.3445 [astro-ph.CO].

[42] J. Hoftuft et. al., Increasing evidence for hemispherical power asymmetry in the five-year WMAP data, Astrophys. J. 699, 985 (2009).

[43] F. K. Hansen et. al., Power asymmetry in cosmic microwave background fluctuations from full sky to subdegree scales: is the Universe isotropic?, Astrophys. J. 704, 1448 (2009).

[44] H. K. Eriksen et. al., Hemispherical power asymmetry in the three-year Wilkinson Microwave Anisotropy Probe sky maps, Astrophys. J. 660, L81 (2007).

[45] H. K. Eriksen et. al., Asymmetries in the CMB anisotropy field, Astrophys. J. 605, 14 (2004). Erratum-ibid. 609, 1198 (2004).

[46] F. K. Hansen, A. J. Banday, and K. M. Gorski, Testing the cosmological principle of isotropy: local power spectrum estimates of the WMAP data, Mon. Not. R. Astron. Soc. 354, 641 (2004).

[47] C. Dvorkin, H. V. Peiris, and W. Hu, Testable polarization predictions for models of CMB isotropy anomalies, Phys. Rev. D 77, 063008 (2008).

[48] K. Land and J. Magueijo, The axis of evil revisited, Mon. Not. R. Astron. Soc. 378, 153 (2007).

[49] K. Land and J. Magueijo, The axis of evil, Phys. Rev. Lett. 95, 071301 (2005).

[50] A. de Oliveira-Costa, M. Tegmark, M. Zaldarriaga, and A. Hamilton, The significance of the largest scale CMB fluctuations in WMAP, Phys. Rev. D 69, 063516 (2004).

[51] D. J. Schwarz, G. D. Starkman, D. Huterer, and C. J. Copi, Is the low-l microwave background cosmic?, Phys. Rev. Lett. 93, 221301 (2004). 
[52] M. Tegmark and A. de Oliveira-Costa, A high resolution foreground cleaned CMB map from WMAP, Phys. Rev. D 68, 123523 (2003).

[53] E. F. Bunn, P. Ferreira, and J. Silk, How anisotropic is our Universe?, Phys. Rev. Lett. 77, 2883 (1996).

[54] M. C. Bento et. al., On the cosmology of massive vector fields with SO(3) global symmetry, Class. Quantum Grav. 10, 285 (1993).

[55] NASA's Wilkinson Microwave Anisotropy Probe homepage: http://wmap.gsfc.nasa.gov/.

[56] A. R. Pullen and M. Kamionkowski, Cosmic microwave background statistics for a direction-dependent primordial power spectrum, Phys. Rev. D 76, 103529 (2007).

[57] ESA's PLANCK mission homepage: http://planck.esa.int/

[58] C. A. Valenzuela-Toledo and Y. Rodríguez, Non-gaussianity from the trispectrum and vector field perturbations, arXiv:0910.4208 [astro-ph.C0].

[59] A. A. Starobinsky, Multicomponent de Sitter (inflationary) stages and the generation of perturbations, Pis'ma Zh. Eksp. Teor. Fiz. 42, 124 (1985) [JETP Lett. 42, 152 (1985)].

[60] M. Sasaki and E. D. Stewart, A general analytic formula for the spectral index of the density perturbations produced during inflation, Prog. Theor. Phys. 95, 71 (1996).

[61] M. Sasaki and T. Tanaka, Superhorizon scale dynamics of multiscalar inflation, Prog. Theor. Phys. 99, 763 (1998).

[62] D. H. Lyth, K. A. Malik, and M. Sasaki, A general proof of the conservation of the curvature perturbation, JCAP 0505, 004 (2005).

[63] D. H. Lyth and Y. Rodríguez, Inflationary prediction for primordial non-gaussianity, Phys. Rev. Lett. 95, $121302(2005)$.

[64] D. H. Lyth, Axions and inflation: vacuum fluctuations, Phys. Rev. D 45, 3394 (1992).

[65] L. Boubekeur and D. H. Lyth, Detecting a small perturbation through its non-gaussianity, Phys. Rev. D 73, 021301(R) (2006).

[66] D. H. Lyth, The curvature perturbation in a box, JCAP 0712, 016 (2007).

[67] Y. Rodríguez and C. A. Valenzuela-Toledo, On the issue of the $\zeta$ series convergence and loop corrections in the generation of observable primordial non-gaussianity in slow-roll inflation. Part II: the trispectrum, arXiv:0811.4092 [astro-ph].

[68] J. Kumar, L. Leblond, and A. Rajaraman, Scale dependent local non-gaussianity from loops, arXiv:0909.2040 [astro-ph.CO].

[69] K. Kohri, D. H. Lyth, and C. A. Valenzuela-Toledo, On the generation of a non-gaussian curvature perturbation during preheating, arXiv:0904.0793 [hep-ph].

[70] E. Komatsu and D. N. Spergel, Acoustic signatures in the primary microwave background bispectrum, Phys. Rev. D 63, 063002 (2001).

[71] J. Maldacena, Non-gaussian features of primordial fluctuations in single field inflationary models, JHEP 0305, 013 (2003).

[72] E. Komatsu et. al., Five-year Wilkinson Microwave Anisotropy Probe (WMAP) observations: cosmological interpretation, Astrophys. J. Suppl. Ser. 180, 330 (2009). 